\section{COLEOPTERA}

\section{New Species of Metachroma}
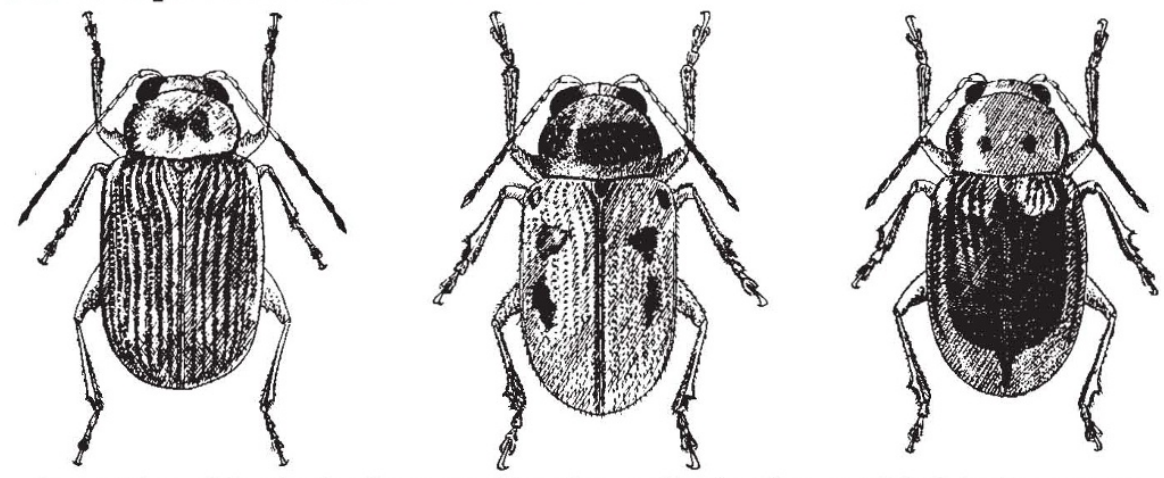

In a review of beetles in the genus Metachroma (family Chrysomelidae) in the western hemisphere, Doris H. Blake describes (Smithson. Contrib. Zool., No. 57; 1970) fortyseven new species, three of which are illustrated here: $M$. ignotum, $M$. hirsutum and $M$. bimarginatum.

\section{POLYPEPTIDES}

\section{Progress on Proinsulin}

from our Molecular Biology Correspondent

THE relation between insulin and its precursor, proinsulin, is now receiving the kind of minute scrutiny that was lavished on the activation of the pancreatic zymogens-a subject about which enough has surely been written to fill a modest library. Bovine proinsulin is a chain of eighty-one residues, of which thirty are excised when it is activated-a much greater fraction of the chain than in any known zymogen-enzyme conversion. Steiner and his colleagues (J. Biol. Chem., 246, 1365; 1971) have now established that the conversion involves more than one proteolytic step, but that the major product is a peptide of twenty-six residues (C-peptide), which remains, together with the insulin, in the pancreatic granules.

The proinsulin fraction as isolated contains two intermediates, one cleaved at the junction of the C-peptide and the insulin A-chain, the other at its junction with the B-chain, and in both cases two amino-acids have been lost. The proinsulin chain can thus be represented, reading from the $\mathrm{N}$-terminus, as B-chain, followed by arg-31 and arg-32, which are lost during activation, followed by the 26-residue C-peptide, then by lys-59 and arg-60-again lost on activation-and finally the A-chain. This remains attached to the B-chain by two disulphide bonds, and doubtless non-covalent interactions. Steiner et al. give a revised sequence for the C-peptide, and show that it is identical with the product of trypsin-cleavage of proinsulin in vitro. The insulin and the $C$-peptide in the pancreatic granules are recovered in equimolar proportion.

The peptide has a curious composition, with a very high content of proline and of glutamic acid. One supposes that the cleavage in situ is effected by trypsin, in just the same way as in vitro, and that the ends of the insulin so liberated are trimmed off by carboxypeptidase B.

In a companion paper Oyer et al. (ibid., 1375) describe the preparation and sequencing of the $C$-peptide from human pancreas. There are not twenty-six but thirty-one residues, and two amino-acids are again lost at either end. A comparison of the sequence with that of the bovine and porcine C-peptides previously determined shows that, making allowance for differences in length, only half the positions are invariant. Because the C-peptide can be presumed to lie on the outside of the molecule, and its removal does not interfere with the packing of side chains in the residual globular insulin molecule, this relatively low degree of conservation, relative to that found in A-
NoBODY has yet come forward with anything approaching a satisfactory explanation of the existence of the muon and the electron although many attempts have been made to prove that the muon is something more than just a heavy version of the electron. It may, however, be that there are "elementary" particles, intimately associated with these two, which have so far managed to evade detection in particle physics experiments.

The next issue of Nature Physical Science includes an article by C. A. Ramm of the European Organization for Nuclear Research (CERN) in which he describes what may be the observation of very short lived particles $\left(<10^{-125}\right)$ related to the muon and electron (all members of the lepton family). Ramm has carried out an analysis of some of the neutrino induced interactions photographed in a heavy liquid bubble chamber at CERN during 196364 and 1967; the ones of particular interest are those in which a muon and a $\gamma$ ray seem to originate from the interaction vertex.

The properties of any group of particles (such as a muon and a $\gamma$ ray) can be described by its invariant mass which is simply a mathematical combination of the total energy and momentum of the group If the muon and B-chains, is perhaps not unreasonable.

Earlier work from the same laboratory showed that refolding of fully reduced denatured proinsulin was an efficient process, by contrast with correct association of separated A- and B-chains, which can be achieved only in relatively low yield. Oyer et al. suggest that therein may lie one of the advantages of the existence of a precursor. One may note in this connexion the recent work of Varandani and Nafz (Arch. Biochem., 141, 533; 1970), who studied the refolding of proinsulin from the "scrambled" form, with the six half-cystine residues randomly paired, under the influence of a refolding enzyme, which catalyses disulphide exchange. They found that the native structure of proinsulin was readily restored (as judged by immunological assay), but native insulin could be regenerated in this system only in the presence of C-peptide (four residues of the complete proinsulin sequence being of course missing). The implication is that the C-peptide binds to insulin, rather in the manner of S-protein and S-peptide in subtilisin-cleaved ribonuclease. Such an interaction is not, however, apparent from the work of Steiner and his colleagues, who find that insulin and C-peptide even circulate independently in the serum. Evidently, conditions for the interaction have to be closely defined if the results of Varandani and $\mathrm{Nafz}$ are to be taken at face value.

\title{
Evidence of Charged Heavy Leptons
}

and $\gamma$ ray produced in the interactions which Ramm has studied were related (in the sense of being the decay products of a very short lived particle), a peak would be expected in the invariant mass spectrum at a value of the mass corresponding to the mass of the new particle.

In fact, Ramm has found just such a peak in the invariant mass curve at about $430 \mathrm{MeV}$. If this peak is genuine -and Ramm produces plenty of evidence to suggest that it is-then the existence of a charged lepton whose mass is about four times that of the muon and eight hundred times that of the electron has definitely been established.

Ramm's conclusion supports his previous proposition of the existence of a heavy neutral lepton with about the same mass (Nature, 227, 1323; 1970) and could also tie in with the work of Liberman et al. (Phys. Rev. Lett., 22, $663 ; 1969)$ who examined the invariant mass distribution of muons and their $\gamma$ ray bremsstrahlung but were unable to make a definitive statement in favour of the existence of heavy leptons. What must now be awaited with interest are the results of experiments which have been carried out at the Stanford Linear Accelerator recently and which could greatly clarify the situation. 